\title{
Extensive air showers' arrival direction distribution by TBS array
}

\author{
Yuri Verbertsky, Manana Svanidze, Abesalom Iashvili, Levan Kakabadze
}

HEP, E Andronikashvili Institute of Physics under Tbilisi State University, Tbilisi, Georgia

Email address:

yuverbetsky@mail.ru (Y. Verbetsky), mananasvanidze@yahoo.com (M. Svanidze)

\section{To cite this article:}

Yuri Verbertsky, Manana Svanidze, Abesalom Iashvili, Levan Kakabadze. Extensive Air Showers' Arrival Direction Distribution by TBS Array. International Journal of High Energy Physics. Vol. 1, No. 4, 2014, pp. 49-54. doi: 10.11648/j.ijhep.20140104.11

\begin{abstract}
Arrival zenith angle distribution for the Extensive Air Showers (EAS) with a wide range of number of charged particles is studied using the experimental data obtained using the EAS 4-detector array TBS in Tbilisi. The station is a part of the GELATICA net in Georgia (GEorgian Large-area Angle and Time Coincidence Array). This experiment is devoted to the study of possible correlations in the arrival times and directions of separate EAS events over large distances and to the Primary Cosmic Ray energy spectrum investigation at very high energies. It is shown that the distribution function with the exponential dependence of showers' flux on absorbing atmospheric depth provides a good approximation for the angular distribution despite the existing azimuth anisotropy of array. The dependence of the EAS absorption path estimation on the angular measure cutoff boundary is studied.
\end{abstract}

Keywords: Extensive Air Showers, Angular Distribution, Resolution Function

\section{Introduction}

The process of Extensive Air Showers (EAS) development in the atmosphere with accompanied absorption manifests itself through the arrival direction distribution. That is why an interest to such investigations is long-standing $[1-6]$. The distribution of zenith angle $\theta$ of the shower arrival direction is usually studied under the assumption of azimuth isotropy for both the Cosmic Ray phenomenon and the measuring equipment.

It has been shown previously [6], that the distribution of zenith angle weakly depends on the energy of Primary Cosmic Ray particles. This feature makes it possible to investigate the subject, even by small installations incapable of EAS energy direct measurement. The data discussed hereafter is obtained by small installation, arranged under the concrete roof of second building of Andronikashvili Institute of Physics in Tbilisi [7 - 9] (EAS goniometer TBS).

\section{Description of the Installation}

The TBS installation includes 4 scintillator detectors controlled by the data acquisition (DAQ) card [10], operating under PC control with a LabView interface for Windows. Detectors are arranged in the corners of a square with $a=10 \mathrm{~m}$ sides; they are arranged under the concrete roof and surrounded by concrete walls. The building is oblong nearly in the East - West direction (Fig. 1). This surrounding is taken into account in the following analysis.

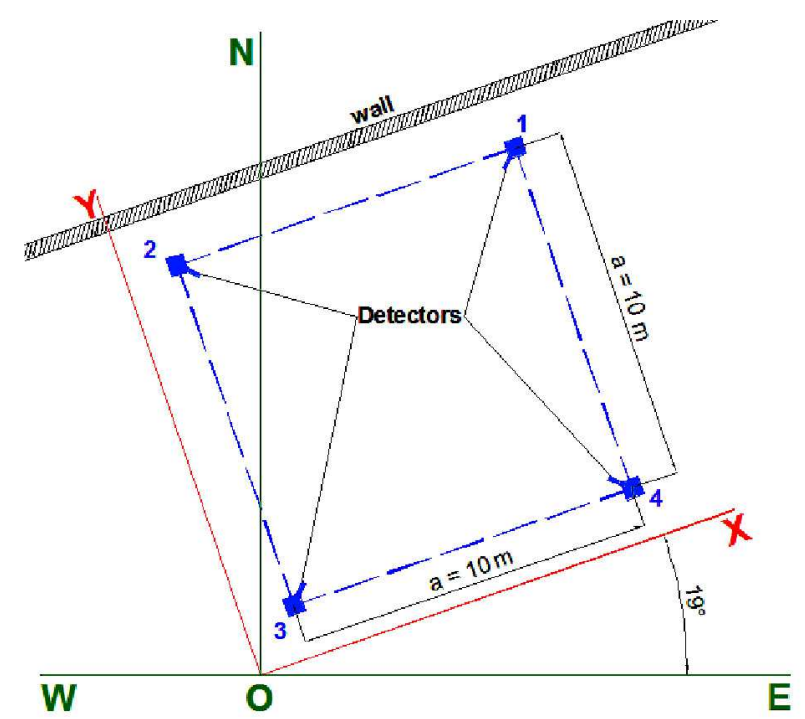

Figure 1. TBS array layout.

Each detector consists of a $5 \mathrm{~cm}$ thick scintillators slab of $(50 \times 50) \mathrm{cm}^{2}$ area, supplied with a photo-multiplier tube (PMT). The PMT pulses, initiated by the passage of EAS 
charged particles through the scintillator material, are read by DAQ card. The equipment measures the pulse delay relative to the 4-fold pulse coincidence with $\tau=1.25 n$ s time slicing step. The data are stored on PC in the form of integer values $k_{1}, k_{2}$, $k_{3}$ and $k_{4}$, corresponding to the numbers of delay slices for every detector. This information allows a posterior estimation of local direction of EAS front arrival [11].

The TBS installation is located at the altitude of $(489 \pm 4) m$ by GPS estimation. The respective estimation of the installation depth in the atmosphere

$$
d_{\mathrm{TBS}}=(976.6 \pm 0.5) \mathrm{g} / \mathrm{cm}^{2}
$$

is derived in accordance with International Civil Aviation Organization standard atmosphere parameters [12]. This value is used for EAS absorption path $\Lambda_{\mathrm{abs}}$ calculation.

\section{Directions Estimation by Square EAS Goniometer}

The TBS installation is a planar EAS goniometer, permitting the linear estimation of the planar (horizontal) components of the unit direction vector of the EAS front's local tangent plane [11]. It is assumed that the front of shower is moving with light velocity $c$.

For the special case of detectors' disposition in the corners of a square (Fig. 1) the estimation of the horizontal 2D projection of this direction vector is

$$
\mathbf{n}=\left(\frac{c \cdot \tau}{2 a}\right)\left(\begin{array}{l}
\left(k_{1}+k_{4}\right)-\left(k_{2}+k_{3}\right) \\
\left(k_{1}+k_{2}\right)-\left(k_{3}+k_{4}\right)
\end{array}\right)
$$

with respect to the XOY reference frame. The dispersion of this vector components' estimation is

$$
\sigma_{x}^{2}=\sigma_{y}^{2}=\left(\frac{c \cdot \tau}{2 a}\right)^{2} \cdot\left(\left(k_{1}+k_{3}\right)-\left(k_{2}+k_{4}\right)\right)^{2},
$$

while the correlation vanishes. Only statistical uncertainty is taken into account.

It is obvious that the measured values of direction vector components (1) possess the magnitudes on the square lattice with step $\delta=c \cdot \tau / 2 a=0.0187$ due to integer values of delay slice numbers $k$.

That is why any EAS event is referable to only one of the lattice sites on the $\left(n_{x}, n_{y}\right)$ plane (Fig. 2), representing some separate area of possible directions on the celestial hemisphere. So the set of these site neighborhoods become the natural bins of 2D histogram.

Fig. 3 shows distribution of arrival direction of 52798 EAS events recorder by TBS. This represents the data analyzed hereinafter.

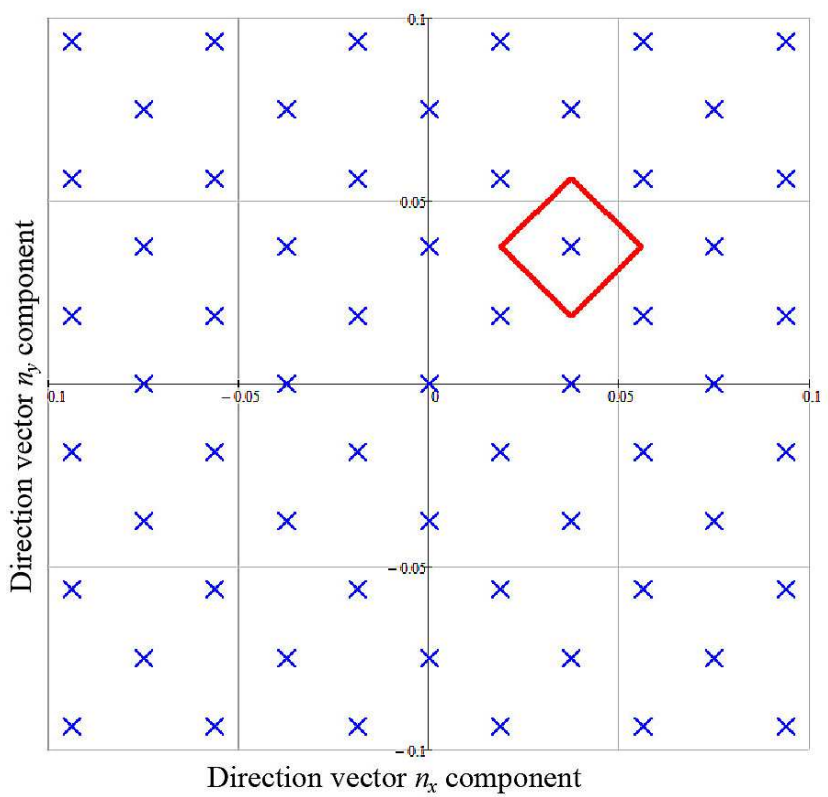

Figure 2. Lattice sites on the $\left(n_{x}, n_{y}\right)$ plane shown in the Zenith projection vicinity. Single bin is marked out in red as an example.

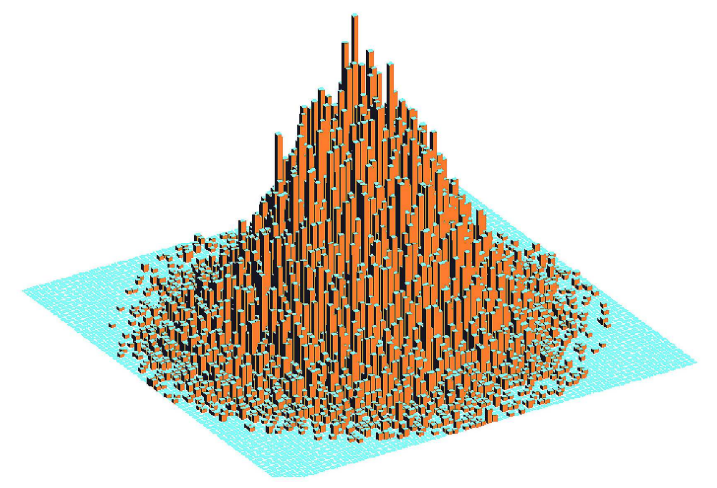

Figure 3. Bar histogram representing EAS arrival intensities. All 52798 events are allocated by bins of $\left(n_{x}, n_{y}\right)$ plane, Fig. 2.

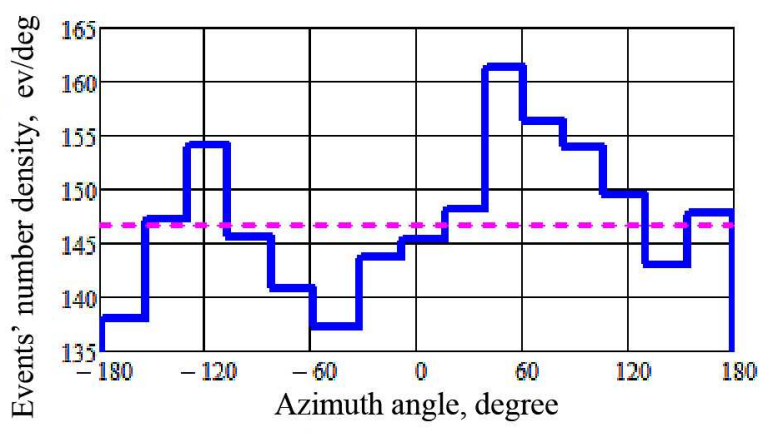

Figure 4. Azimuth angle distribution density histogram by TBS data. Dash line represents correspondent isotropic distribution.

As shown in Fig. 4, the arrival azimuth direction distribution is rather asymmetric due to the existing asymmetry of detectors' surroundings. This asymmetry must be taken into account as a correction to the fundamentally symmetric distribution. Fig. 4 also indicates that the dominant harmonic of existing azimuth anisotropy has a semi-circle length of period. 
Arrival zenith angle distribution is shown in Fig. 5. The following analysis and estimations are based on these data.

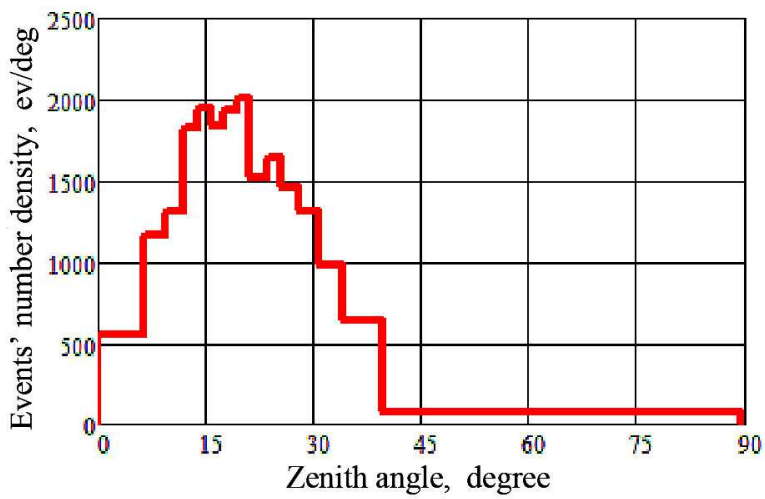

Figure 5. Zenith angle distribution density histogram by TBS data.

\section{Considered Form of Distribution}

\subsection{Fundamental Distribution of Zenith Angle}

We shall assume that all EAS developed in the atmosphere are absorbed in compliance with the usual exponential low [6]. Thus the flux of EAS observed in the unit solid angle by the installation located under the atmospheric layer of depth $d$ is proportional to

$$
\exp \left\{-\left(d / \Lambda_{\mathrm{abs}}\right) \cdot \sec (\theta)\right\}
$$

Here $\Lambda_{\mathrm{abs}}$ is the EAS absorption path required. The dependence is assumed to be applicable in the framework of a flat atmospheric model, i.e. in the interval $0 \leq \theta \leq 60^{\circ}-70^{\circ}$ of zenith angles. Taking into consideration that the TBS goniometer employs the flat detectors located in the horizontal plane (i.e. adding a $\cos (\theta)$ factor to (3)), let us integrate the observed flux expression by the azimuth to get a zenith angle distribution in the form of

$$
f_{\theta}(\theta) \sim \sin (\theta) \cos (\theta) \cdot \exp \left\{-\left(d / \Lambda_{\mathrm{abs}}\right) \cdot \sec (\theta)\right\} .
$$

As is mentioned above, the planar goniometers are capable of straight estimation of two components of EAS arrival direction unit vector only, i.e. $n_{x}, n_{y}$, being parallel to the detectors' location plane [11]. That is why the immediate variable, independent of any additional assumption and measuring the event separation from the zenith direction is the estimated length of the unit direction vector projection onto the detectors' plane

$$
\alpha=\sqrt{n_{x}^{2}+n_{y}^{2}}=\sin (\theta), \quad 0 \leq \alpha \leq 1 .
$$

This variable gives estimate of the usual zenith angle indirectly. The distribution of EAS arrival zenith angle (4) expressed in the terms of this new variable proves to be the function:

$$
f_{\alpha}(\alpha \mid q)=\frac{\alpha \cdot \mathrm{e}^{-\frac{q}{\sqrt{1-\alpha^{2}}}}}{\int_{0}^{1} \alpha \cdot \mathrm{e}^{-\frac{q}{\sqrt{1-\alpha^{2}}}} d \alpha}
$$

Here value $q=d / \Lambda_{\mathrm{abs}}$ measures the EAS vertical path range. The fundamental distribution function (6) has to be corrected to take into account the distortions by the surrounding matter anisotropy and installation's resolution function before the comparison with TBS data in the following.

\subsection{Anisotropy Account}

All arrival direction data is divided by intervals of the zenith angle for the purpose of the study of existing anisotropy. The polar histograms are constructed in every interval. They are approximated by angular distributions of the form:

$$
P(\varphi)=\frac{1+2 b \cdot \cos \left(2\left(\varphi-\varphi_{0}\right)\right)}{2 \pi} .
$$

Here the above mentioned observation that the dominant harmonic of existing azimuth anisotropy has a semi-circle length of period is used. The initial phase parameter $\varphi_{0}$ determines the direction of the azimuth distribution maximum, while the amplitude $b$ measures a difference with the isotropic distribution $(1 / 2 \pi)$. One of the approximated distributions is shown in the Fig. 6 as an example. In this particular case the anisotropy amplitude estimation value is $b=0.058 \pm 0.011$.

In accordance with angular distribution (7), we attempt to account for the influence of the azimuth-anisotropic surroundings (i.e. the building walls etc.) by adding to the exponent index in (6) of form

$$
\frac{B(\alpha)}{\sqrt{1-\alpha^{2}}} \cos 2\left(\varphi-\varphi_{0}(\alpha)\right)
$$

Here the function $B(\alpha)$ allows to account for the additional matter of the surroundings reduced to the horizontal layer. The initial phase of anisotropy $\varphi_{0}(\alpha)$ depends on the zenith distance parameter $\alpha$.

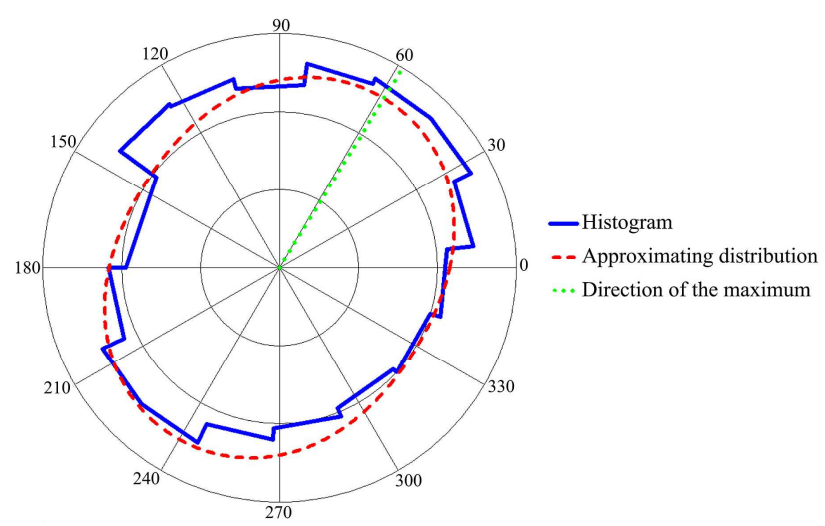

Figure 6. Polar plot of EAS events' azimuth distribution for zenith angle interval $30^{\circ}<\theta<40^{\circ}$ 
The anisotropy existing is shown in the Fig. 7. It is approximated with a function of the form

$$
B(\alpha)=p \cdot\left(\mathrm{e}^{k \alpha}-1\right),
$$

shown in the figure, too.

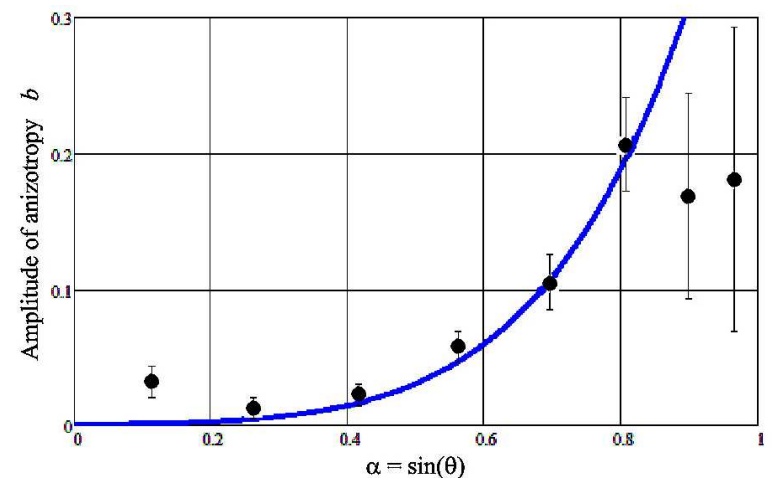

Figure 7. The amplitude $B(\alpha)$ of existing azimuth anisotropy.

The distribution function, corrected for the anisotropy distortions (8) and (9), is defined to be proportional to the integral:

$$
\begin{aligned}
& \int_{0}^{2 \pi} \alpha \cdot \mathrm{e}^{\frac{-q+p \cdot(\exp (k \alpha)-1) \cos 2\left(\varphi-\varphi_{0}(\alpha)\right)}{\sqrt{1-\alpha^{2}}} d \varphi} \\
& =\alpha \cdot \exp \left\{-q / \sqrt{1-\alpha^{2}}\right\} \cdot \frac{\pi}{2} \mathrm{I}_{0}\left(\frac{p \cdot\left(\mathrm{e}^{k \cdot \alpha}-1\right)}{\sqrt{1-\alpha^{2}}}\right) .
\end{aligned}
$$

Here the integral representation of modified Bessel function is used. Obviously, any dependence on the initial phase $\varphi_{0}(\alpha)$ is dropped out due to full period integration.

So, the azimuthally distorted distribution density is:

$$
f_{\mathrm{I}}(\alpha \mid q, p, k)=\frac{\alpha \cdot \exp \left\{-q / \sqrt{1-\alpha^{2}}\right\}}{N(q, p, k)} \cdot \mathrm{I}_{0}\left(\frac{p \cdot\left(\mathrm{e}^{k \cdot \alpha}-1\right)}{\sqrt{1-\alpha^{2}}}\right)
$$

The normalizing factor is defined as usual integral:

$$
N(q, p, k)=\int_{0}^{1} \alpha \cdot \exp \left\{-q / \sqrt{1-\alpha^{2}}\right\} \cdot \mathrm{I}_{0}\left(\frac{p \cdot\left(\mathrm{e}^{k \cdot \alpha}-1\right)}{\sqrt{1-\alpha^{2}}}\right) d \alpha
$$

It can be seen from (10) that full influence of azimuth anisotropy in the used approximation is represented by the factor $\quad \mathrm{I}_{0}\left(p \cdot\left[e^{-k \alpha}-1\right] /\left[1-\alpha^{2}\right]^{1 / 2}\right), \quad$ containing both apparatus-specific parameters $p$ and $k$.

Just the free parameters $q, p, k$ have to be obtained from the distribution (10) in comparison with observed data, accounting the resolution function of the installation.

\subsection{Resolution Function}

Detectors of TBS installation are located symmetrically at the vertices of a square. The estimations of components of
EAS arrival direction vector are uncorrelated and equal-dispersion (2) in this case. The estimations are obtained by means of linear transformation (1) of directly measured integer timing $k$ numbers of signals' from the detectors. Therefore it is possible to use the assumption that the joint distribution of estimates of $n_{x}, n_{y}$ components can be approximated by the Normal distribution, with the dispersion matrix D proportional to identity:

$$
\begin{aligned}
G\left(\mathbf{n} \mid \mathbf{n}_{0}, \sigma\right) \sim \exp \left\{-\frac{1}{2}\left(\mathbf{n}-\mathbf{n}_{0}\right)^{\top} \cdot \mathbf{D}^{-1} \cdot\left(\mathbf{n}-\mathbf{n}_{0}\right)\right\} ; \\
\mathbf{n}=\left(\begin{array}{l}
n_{x} \\
n_{y}
\end{array}\right) ; \quad \mathbf{D}=\left(\begin{array}{cc}
\sigma^{2} & 0 \\
0 & \sigma^{2}
\end{array}\right) ;
\end{aligned}
$$

Here $\mathbf{n}_{0}$ vector represents the position of true direction (unknown). Only $\mathbf{n}$ vector may be measured, with D uncertainty, of course.

Let us integrate this distribution by azimuth to obtain the radial distribution needed. While $\alpha$ is a distance from Zenith point to the direction measured (5), and $\varphi=\arctan \left(n_{y} / n_{x}\right)$ is the correspondent azimuth angle, we get

$$
\frac{\left(\mathbf{n}-\mathbf{n}_{0}\right)^{\top} \mathbf{D}^{-1}\left(\mathbf{n}-\mathbf{n}_{0}\right)}{2}=\frac{\alpha_{0}^{2}+\alpha^{2}-2 \alpha \alpha_{0} \cos \left(\varphi-\varphi_{0}\right)}{2 \sigma^{2}} .
$$

The azimuth integral of the correspondent exponent is:

$$
\int_{0}^{2 \pi} \mathrm{e}^{-\frac{\alpha_{0}^{2}+\alpha^{2}-2 \alpha \alpha_{0} \cos \left(\varphi-\varphi_{0}\right)}{2 \sigma^{2}}} d \varphi=2 \pi \mathrm{e}^{-\frac{\alpha_{0}^{2}+\alpha^{2}}{2 \sigma^{2}}} \cdot \mathrm{I}_{0}\left(\frac{\alpha \alpha_{0}}{\sigma^{2}}\right) \text {. }
$$

It is handy to define a scaled Bessel function $\mathrm{I}_{0}{ }^{*}(x)=\mathrm{e}^{-x} \mathrm{I}_{0}(x)$ to get useful form of expressions.

Consequently the resolution function, i.e. conditional distribution of $\alpha$ variable estimation (measuring the distance of EAS event's arrival direction from the Zenith point) under the assumption, that true value of this distance is $\alpha_{0}$, can be defined under account of $0 \leq \alpha \leq 1$ constraint as:

$$
R\left(\alpha \mid \alpha_{0}, \sigma\right)=\frac{\alpha \cdot \mathrm{e}^{-\frac{\left(\alpha_{0}-\alpha\right)^{2}}{2 \sigma^{2}}} \cdot \mathrm{I}_{0}^{*}\left(\alpha \cdot \alpha_{0} / \sigma^{2}\right)}{\int_{0}^{1} \mathrm{e}^{-\frac{\left(\alpha_{0}-\alpha\right)^{2}}{2 \sigma^{2}}} \cdot \mathrm{I}_{0}^{*}\left(\alpha \cdot \alpha_{0} / \sigma^{2}\right) \cdot \alpha d \alpha} .
$$

So, it is necessary to employ some previously obtained estimation of $\alpha$-values standard deviations.

The observed data allows determination of the standard deviation dependence on the event's distance from Zenith. As shown in Fig. 8, one can use the following approximation:

$$
\begin{array}{cc}
\Sigma(\alpha)=\sigma_{0}+\sigma_{1} / \sqrt{1-\alpha^{2}} ; & \sigma_{0} \approx 7.3 \cdot 10^{-2} . \\
\left(=\sigma_{0}+\sigma_{1} \cdot \sec (\theta)\right) & \sigma_{1} \approx 6.1 \cdot 10^{-3} .
\end{array}
$$

Now the resolution function is completely defined. Some examples of this function are shown in Fig. 9. 


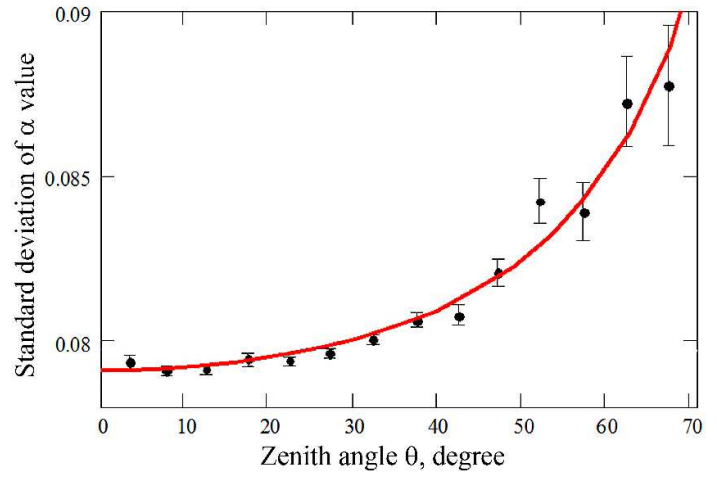

Figure 8. Dependence of standard deviations of $\alpha$ variable estimation value on zenith angle.

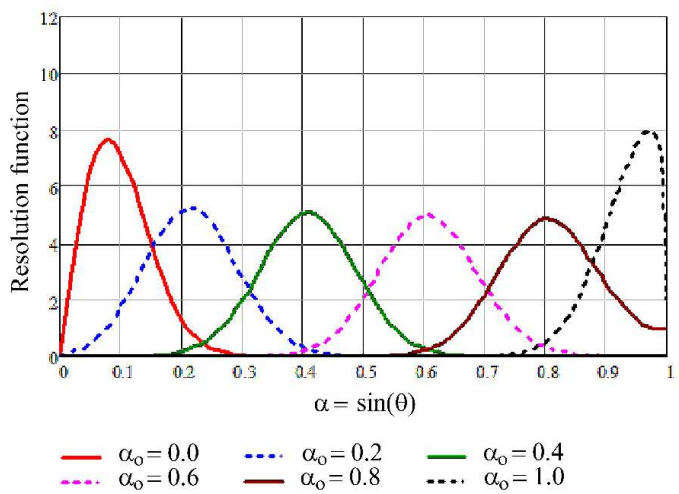

Figure 9. Resolution function for some values of true zenith distance $\alpha_{0}$.

The definitions (10), (11) now allow to construct the final probability density function of the event's observed distance $\alpha$ from Zenith:

$$
\begin{aligned}
& f_{\mathrm{II}}(\alpha \mid q, p, k)= \\
& =\int_{0}^{1} R\left(\alpha \mid \alpha_{0}, \Sigma(\alpha)\right) \cdot f_{\mathrm{I}}\left(\alpha_{0} \mid q, p, k\right) d \alpha_{0} .
\end{aligned}
$$

Standard deviation dependence on $\alpha$ value (12) is used here.

This distorted density function is the one to be compared with the TBS observed data to estimate the apparatus-specific parameters $p, k$ and the EAS absorption path $\Lambda_{\text {abs }}$ required.

\section{Estimation of Parameters}

At first let us estimate the value of empiric parameters $p$ and $k$, which generally describe the anisotropic properties (8), (9), of the matter surrounding the installation. The method of maximum likelihood has been used for this purpose, applied to the part of the data in the angular interval of $0 \leq \theta \leq 70^{\circ}$, i.e. in the range of applicability of the flat atmosphere model. While provisionally disregarding the $q$ value estimation, we get the apparatus-specific parameters' estimations for the surroundings description

$$
p=(14.5 \pm 0.5) 10^{-3}, \quad k=6.0 \pm 0.2,
$$

with the correlation coefficient being negligible.
In order to estimate the required EAS absorption path $\Lambda_{\mathrm{abs}}=d_{\mathrm{TBS}} /(q-p)$ let us investigate dependence of the $q$ value estimation on the position of the upper trimming limit $\alpha_{\text {trim }}$ of data subset used. The correspondent sequence of $\Lambda_{\text {abs }}$ estimations is obtained by repeatedly applying the maximum likelihood method to the sequentially expanding data subsets. The resulting dependence is shown in Fig. 10. (All points in this sequence of estimations are mutually dependent!)

As can be seen from the Fig. 10, these estimations become stable within one standard deviation width for the trimming limits within the interval of $0.550 \leq \alpha_{\text {trim }} \leq 0.875$. That is why we adopt the final estimation of EAS absorption path

$$
\Lambda_{\mathrm{abs}}=(115.4 \pm 2.6) \mathrm{g} / \mathrm{cm}^{2}
$$

corresponding to the maximal trimming limit of the interval of stability. It is valid within the interval $0 \leq \alpha \leq 0.875$ of event's distance from Zenith, therefore.

Disagreement between the distribution (13) and data quickly increases beyond the domain of applicability of a flat atmosphere model. It can be seen in Fig. 11 where the data points deviate appreciably from the optimal distribution curve for zenith angles overriding the actual limit of the model validity range $\theta_{\text {lim }}=62^{\circ}$, i.e. $\alpha_{\text {lim }}=0.883$.

Both surrounding's anisotropy and installation's resolution broadens the distribution of existing data as compared with correspondent physical distribution.

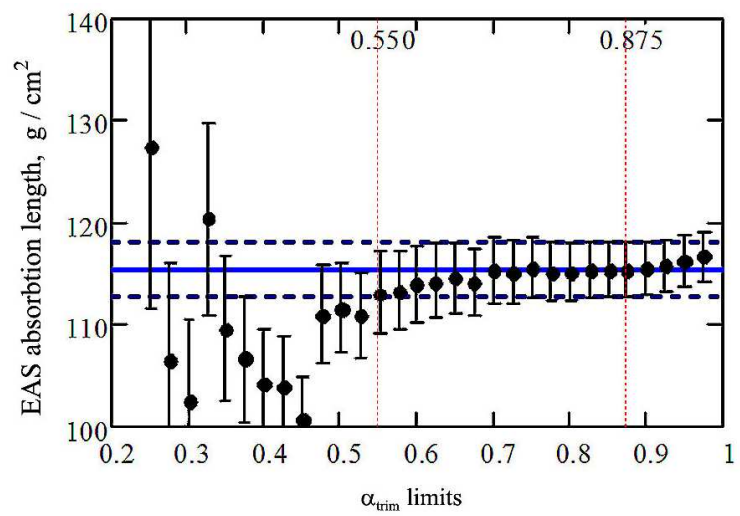

Figure 10. Dependence of EAS absorption pass estimation on the $\alpha$-trimming limit.

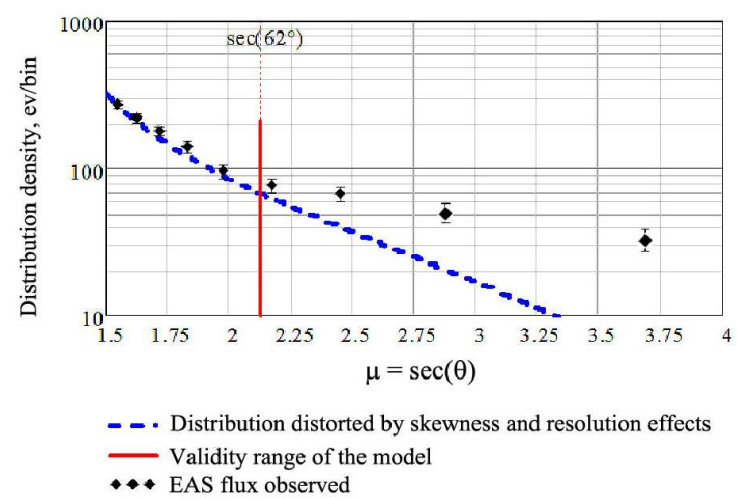

Figure 11. Discrepancy in the horizon vicinity between TBS measured data and distorted distribution (13) fitted. 


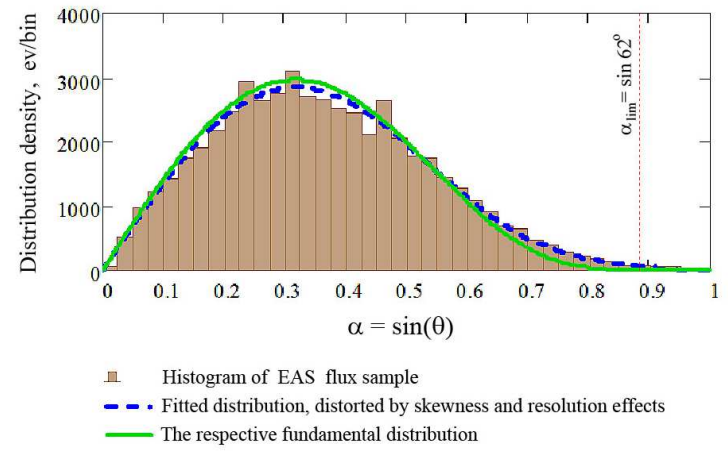

Figure 12. Comparison of the observed data and distributions obtained.

The key influence of these distorting impacts is explicitly expressed in the difference of the fundamental distribution (6) and fitted distorted one (13), shown in Fig. 12. Any incautious attempt of immediate fitting of fundamental distribution to the existing data results in unstable estimation of $\Lambda_{\mathrm{abs}}$, not in agreement with existing previous world data.

\section{Conclusions}

It has been established that the account for the resolution function and azimuth anisotropy of TBS installation surroundings makes it possible to employ the fundamental distribution (6) of event's distance from Zenith $\alpha=\sin (\theta)$. This model of regular EAS absorption, in accordance with flat atmosphere model approximation, has proved to be valid for description of EAS absorption process within the interval $0 \leq \alpha \leq 0.883$ of event's distance from Zenith, i.e. in the interval $0 \leq \theta \leq 62^{\circ}$ of zenith angle. The estimated value of EAS absorption path is actually stable under variation of data trim upper limits within $0.550 \leq \alpha_{\text {trim }} \leq 0.875$ and is fixed at the magnitude $\Lambda_{\mathrm{abs}}=(115.4 \pm 2.6) \mathrm{g} / \mathrm{cm}^{2}$. Any estimation of this parameter upon the more restricted sequence of intervals of $\alpha$ variable is unstable. It is the immediate consequence of this study, that any attempt of absorption path estimation with use of some data trimming, not proved to be consistent with stability under variation of this trimming limit, is unreliable, indeed. Our $\Lambda_{\text {abs }}$ estimation is in approximate agreement with the previous estimations $[1,2,5$, and 6] by installations located at low altitudes.

\section{Acknowledgements}

The authors are grateful to other current and former members of our group for their technical support. We are especially thankful to our colleagues working now in foreign research centers for their permanent interest in our investigations.

This work was supported by the Georgian National Science Foundation subsidy for a grant of scientific researches \#GNSF/ST06/4-075 (№ 356/07) and by Shota Rustaveli National Science Foundation, Project \#DI/6/6-300/12.

\section{References}

[1] Greisen K. "Progress in Cosmic Ray Physics," (J. Wilson, ed.) V. 3 p 7. Moscow, (1958) [Russian translation].

[2] D. Ciampa, R.W. Clay, "The zenith angle distribution of extensive air showers at sea level: A measure of shower development", J. Phys. G14 (1988) pp 787-792.

[3] Bejl P.F., Bejsembaev R.U., Vildanov N.G., G.Ya. Goryacheva, V.V. Zhukov et al., "Angular Distribution of EAS at $\mathrm{N}>10^{7}$ particles" Proc. 28th ICRC. Tsukuba. (2003). 1. p 9-12

[4] Yakovlev V.I., Izv. RAN. Ser. fiz. (2004), V68. p 1630.

[5] A. Iyono, C. Noda, H. Matsumoto, M. Masuda, T. Wada, et al., "Zenith Angle Distribution and Atmospheric Effect for EAS with LAAS experiments" Proc. 30th ICRC Mexico (2008), Vol. 4 (HE part 1), pp 47-50

[6] M.Yu. Zotov, N.N. Kalmykov, G.V. Kulikova, V.P. Sulakov., "On the angular distribution of extensive air showers" arXiv:0910.4298v1 [astro-ph.HE] (2009), pp 1-5

[7] Svanidze Manana, Verbetsky Yuri, Bagaturia Yuri, Javrishvili Ala, Eristavi Neli et al., "Investigation of Extensive Air Showers by means of the Network of the Cosmic Rays Stations in Georgia" GESJ: Physics (2010) No.1(3) pp 14-24.

[8] Manana Svanidze, Yuri Verbetsky, Yuri Bagaturia, Ala Javrishvili, Abesalom Iashvili et al., "First results obtained by the GELATICA Network of the Cosmic Rays Stations in Georgia" GESJ: Physics (2011) No.1(5) pp 84-96.

[9] Svanidze Manana, Verbetsky Yuri, Tskhadadze Edisher, Iashvili Abesalom, Kokorashvili Davit. "Some properties of two cosmic ray stations appertained to the GELATICA Network in Georgia" Proc. 32nd ICRC Beijing (2011) pp 1-5

[10] Hansen S, Jordan T, Kiper T, Claes D, Snow G, et al., "Low Cost Data Acquisition Card for Network Cosmic Ray Detector", IEEE Trans. Nucl. Sci., (2004), V51, issue 3, pp 926-930

[11] Svanidze M.S., Verbetsky Yu.G., "Improved method of the Extensive Air Shower arrival direction estimation" arXiv:0804.1751 [astro-ph] (2008) pp 1-13

[12] "Manual of the ICAO standard atmosphere", International Civil Aviation Organization, (1993). 